\title{
Die mittelägyptischen Nisben als Nuklei in präpositionaler, limitierender
} und Genitiv - Relation

D IESER Aufsatz soll eine systematische Abgrenzung verschiedener Nisbe-Konstruktionen des Mittelägyptischen bringen; genau genommen gelten die Regeln mehr oder minder für die älteste ägyptische Schriftsprache, die sich vom "Altägyptischen » über das "Mittelägyptische» bis zum «Spätägyptischen» erstreckt, in ihrer Gesamtheit. Der unglückliche Terminus "Mittelägyptisch » steht in der Überschrift, weil die Darstellung dieser Schriftsprache vorwiegend unter dieser Kennmarke zu laufen pflegt, und weil die folgende Beschreibung das sog. Mittelägyptisch im besonderen betrifft $\left({ }^{(}\right)$.

Behandelt werden die zweigliedrigen Syntagmen, in denen Nisben als erstes Glied stehen. Als zweites Glied kommen nur Substantive vor, wobei unter Substantiv nicht bestimmte Bildungen aus der Formenlehre zu verstehen sind, sondern eine Funktionswortart, die "substantivierte » und andere (teil)funktionsgleiche Elemente, wie z. B. das Suffixpronomen, mitumfasst. In allen Fällen ist das zweite Glied, der Satellit, dem' ersten, dem Nukleus, subordiniert; das Substantiv hängt von der Nisbe ab.

Die Abhängigkeit des substantivischen Satelliten von der Nisbe wird von den Grammatikern des Ägyptischen verschieden beurteilt. Drei Konstruktionsweisen werden ins Feld geführt $\left({ }^{2}\right)$ :

1 Das Substantiv hängt von der Nisbe so ab wie ein Substantiv von einer Präposition. Diese Abhängigkeitsrelation bezeichne ich als "präpositionale Relation » $\left({ }^{3}\right)$.

(1) Wie die ägyptisch-koptische Sprachgeschichte tatsächlich einzuteilen ist, und wie wenig die strenge Scheidung zwischen "Alt- " und "Mittelägyptisch " berechtigt ist, hat H. Stricker, De indeeling der Egyptische taalgeschiedenis, 1945, dargelegt ; die detaillierte Beschreibung des sog. Altägyptischen in E. Edels Grammatik, die einen eingehenderen Vergleich der beiden Sprachstufen erlaubt, stösst die Quintessenz der Strickerschen Arbeit nicht um.

(2) Für die Einzelnachweise s. W. Westendorf, "Hiess Lamares Lamares? ", MIO 7 (1960), $316 \mathrm{f}$.

(3) Die "präpositionale Relation " ist streng getrennt zu halten von der " adver- 
2 Das Substantiv ist ein "bestimmendes Substantiv 》 wie in der Verbindung "Adjektiv + bestimmendes Substantiv 》. Diese Abhängigkeitsrelation nenne ich "limitierende Relation" (oder kurz "Limitation »).

3 Nisbe + Substantiv ist eine (direkte) "Genitiv »verbindung. Diese Abhängigkeitsrelation soll "Genitivrelation " heissen (1).

Im grossen und ganzen geht die Absicht dahin, e i n e dieser drei Möglichkeiten mit Ausschluss der beiden andern als gültig zu erweisen. Diese Vorentscheidung ist nicht ganz richtig, weil, wie noch darzulegen, alle drei Konstruktionen existieren. Sie ist aber zunächst nebensächlich, da man dabei überhaupt nur die Bestimmung der häufigsten Relation ins Auge fasst. Mit ihr soll daher die Diskussion beginnen.

\section{Relationsnisben in präpositionaler Relation.}

Die häufigste und typischste Konstruktion ist vom Typ $t p . j \underline{d} w(. f)$ " der auf (seinem) Berg (Befindliche). Die Nukleusstelle ist mit einer von einer Präposition abgeleiteten Nisbe besetzt, die wie die Präposition eine Lagebestimmung beinhaltet. Diese Gruppe von Nisben, die sich inhaltlich und in ihrer Funktion von den meist von Nomina abgeleiteten "Qualitätsnisben " abhebt, soll "Relationsnisben " genannt sein $\left({ }^{2}\right)$.

Die Erklärung als "Adjektiv + bestimmendes Substantiv》 (limitierende Relation) hat W. Westendorf mit gutem Grund ausgeschieden $\left(^{(3)}\right.$ : in einer solchen Konstruktion dürfte der Satellit nicht wieder

bialen Relation ", die ich andernorts einführe : in der "adverbialen " ist der Nukleus ein Nomen, in der präpositionalen eine Präposition. In $m w$ m jtr. $w$ " das Wasser im Fluss " z. B. kann die Relation zwischen $m w$ und $m$ die "adverbiale" sein, die zwischen $m$ und $j t r . w$ ist die "präpositionale ".

(1) Den Terminus "Genitivrelation " möchte ich dem sog. direkten Genitiv vorbehalten; für den indirekten, der ein formales Kennzeichen, die ursprüngliche Nisbe $n . j$, hat, ist eine Bezeichnung nach der Form möglich, z. B. "nj-Relation ".

(2) "Relation " (I) ist, um das ausdrücklich zu sagen, im Wort "Relationsnisbe " natürlich etwas ganz anderes als in den "Relationen "(II) der Syntagmen (z. B. präpositionale Relation). Auch eine Qualitätsnisbe, die in Opposition zu Relationsnisben steht, tritt in syntaktische "Relationen " (II) ein. "Relation " II bezieht sich auf Syntagmen, "Relation" I als Inhaltsbeschreibung auf eine Wortklasse. Der Tatbestand ist so klar und der systematische Ort der beiden Termini in der Grammatik so verschieden, dass man nicht auf eine deutsche Terminologie wie " lagebestimmende Nisbe » oder "Lagenisbe » für " Relation » I auszuweichen braucht. - Zur Präzisierung der "Relationsnibe"s. unten.

(3) A. a. O., 317. 
selbst Nukleus sein, dürfte also auch kein Suffixpronomen (wie in $\underline{d} w . f)$ nach sich haben.

Dagegen ist W. Westendorfs Entscheidung zugunsten eines "Genitivs» nicht stichhaltig. Für eine "Genitiv "verbindung spricht nach seiner Meinung «die Ableitung [der Nisbe] von der Präposition die ebenfalls eine Genitivverbindung mit dem von ihr regierten Wort eingeht; dafür spricht ferner das Auftreten der Possessivsuffixe bei pronominalem Gebrauch » $\left.{ }^{1}\right)$. Der Schluss, dass das Verhältnis zwischen Nisbe und Nomen dasselbe ist wie das zwischen Präposition und Substantiv, leuchtet ein. Doch handelt es sich bei der Verbindung einer Präposition und eines Substantivs überhaupt um einen "Genitiv»? Was ist schliesslich ein «Genitiv »? Der sog. direkte Genitiv des Ägyptischen, um den es sich dreht, ist ja nicht, wie ein Kasus, einer engen Liste morphologischer Merkmale zugeordnet $\left({ }^{2}\right)$, sondern zunächst einmal nur ein bestimmtes gegenseitiges Verhältnis zweier Glieder eines Syntagmas, eine Relation. Für das Bestehen dieser Relation kann man dann natürlich bis $\mathrm{zu}$ einem gewissen Grad äussere Bedingungen angeben, so vor allem die Besetzung der Planstellen durch bestimmte, nicht zuletzt auch morphologisch vielfach gekennzeichnete Wortklassen. Nun wird jede Definition des sog. direkten Genitivs darauf abheben, dass die Nukleusstelle mit einem Substantiv oder allgemeiner: mit einem Nomen besetzt ist. Die Präpositionen sind aber im Mittelägyptischen keine Nomina. Der morphologische und funktionelle Unterschied ist derart gross, dass eine Vereinbarung beider unter ein er Wortklasse nicht akzeptabel ist ; sie ist wohl auch von niemand je gefordert worden. Dass Präpositionen in Urzeiten einmal Nomina w a r e $\mathrm{n}$, und dass das Verhältnis zwischen solchen Präpositionen und dem Satelliten deshalb einmal ein sog. direkter Genitiv w a r, ist für die $\mathrm{Be}-$ schreibung der syntaktischen Verhältnisse des Mittelägyptischen nicht ausschlaggebend. Der sog. direkte Genitiv hat sich eben in zwei Relationen aufgespaltet. Es bleibt danach nur die Möglichkeit, neben dem sog. direkten Genitiv eine eigene "präpositionale Relation » anzusetzen $\left({ }^{3}\right)$.

(1) loc. cit.

(2) Ein lautliches Kennzeichen gewöhnlicher "Genitiv "verbindungen kann der Status constructus des Nomen regens sein ; dazu ist die Opposition zu einem Status absolutus notwendig. Bei Präpositionen fehlt eine solche Opposition und damit das Kennzeichen.

(3) Letztlich ist der Grund für die Scheidung der beiden Relationen natürlich 
Schwächer noch ist das zweite Argument, die Verbindung der Nisbe mit dem Suffixpronomen. Das Suffixpronomen als Satellit steht nämlich nicht nur in den Verbindungen mit Substantiv und in denen mit Präposition - diese beiden Fälle gehören nach dem Gesagten zwei verschiedenen Relationen an -, sondern auch in der Suffixkonjugation. Der historische, vermutete "Genitiv " (oder was es immer gewesen sein mag) ist hier im Laufe der Sprachgeschichte zu einer "prädikativen Relation " geworden, die mit einem "Genitiv " in synchronischem Zusammenhang nicht das geringste mehr zu tun hat. Das Suffixpronomen ist im Mittelägyptischen nicht auf eine einzige Funktion festgelegt; deshalb lassen sich an ihm allein auch keine Funktionen ablesen. So darf man auch ohne weiteres das Suffixpronomen nach einem Nomen und das Suffixpronomen nach einer Präposition auf verschiedene Funktionen verteilen.

Schliesslich als letztes ein drittes Argument, das W. Westendorf in seiner übersichtlichen Diskussion nicht eigens ins Feld führt, aber doch nennt: der Wechsel der Konstruktion Nisbe + Substantiv mit der Konstruktion Nisbe $+n . j+$ Substantiv, d. h. mit dem sog. indirekten Genitiv. Dazu ist nur eines zu sagen: der sog. indirekte Genitiv drängt im Laufe der Sprachgeschichte verschiedene syntaktische Relationen zurück, also nicht nur den sog. direkten Genitiv (1).

nicht die Verschiedenheit der Wortarten des Nukleus, sondern der, dass man beide Relationen nicht ohne Verrenkungen auf einen gemeinsamen inhaltlichen Nenner bringen kann, oder doch der, dass ein solcher gemeinsamer Nenner bei der systematischen Beschreibung alle r syntaktischen Relationen keinen effektiven Vorteil bringt.

(1) So tritt der sog. indirekte Genitiv an die Stelle des "adjektivischen Attributs " (Erman, NäG $\mathrm{N}^{2}$ § 225). Genauer ist es wohl so : das "Adjektiv " wird zwar häufig einfach durch $n+$ "Adjektiv" ersetzt; zum guten Teil aber auch zum Nukleus des mit $n$ angeschlossenen, ihm untergeordneten Substantivs umgeprägt. An der späteren Beliebtheit des sog. indirekten Genitivs als Ersatz älterer "synthetischer "Relationen ändert die letzte Version nichts. Vgl. weiter die Veränderungen in der Konstruktion der Zahlwörter (K. Sethe, Von Zahlen und Zahlworten, passim). - Der sog. indirekte Genitiv ist - vielleicht abgesehen von dreigliedrigen Nisbekonstruktionen vom Typ $X$ tp.j $Y$ «der auf $\mathrm{Y}$ befindliche $\mathrm{X}$ 》, die ja ebenso strukturiert sind oder waren wie der sog. indirekte Genitiv - die einzige "analytische " Relation des mittelägyptischen Wortgruppenbaus mit nominalem $\mathrm{Nu}$ kleus (Die Präpositional- und Adverbialattribute, die wohl erst im Kommen sind, lasse ich dabei unbeachtet). Es nimmt daher nicht wunder, wenn gerade diese Relation sich unter der allgemeinen "analytischen " Tendenz des Ägyptischen (F. Hintze, "Die Haupttendenzen der ägyptischen Sprachentwicklung ", Zeitschrift 
Das Eintreten eines sog. indirekten Genitivs bedeutet daher nicht in jedem Fall, dass ein sog. direkter Genitiv ersetzt würde.

Es ergibt sich somit, dass die gewöhnliche Verbindung zwischen Relationsnisbe und Substantiv der zwischen Präposition und Substantiv entspricht, und dass hier eine "präpositionale Relation " vorliegt, kein "Genitiv.».

\section{Qualitätsnisben in limitierender Relation (Limitation).}

Nisben sind durch ableitende Wortbildung in die Klasse der "Adjektive " überführte Nomina und Präpositionen. Was es mit den «Adjektiven» des Ägyptischen auf sich hat, lasse ich hier einmal beiseite ; für den gegenwärtigen Zweck sind die Auffassungen der Grammatiken hinreichend zutreffend. Während in die in Abschnitt 1 behandelte präpositionale Relation naturgemäss nur die von Präpositionen abgeleiteten Relationsnisben eingehen können, ist die Konstruktion der "Adjektive " mit bestimmendem Substantiv, die limitierende Relation oder Limitation, dem "adjektivischen " Charakter a lle r Nisben nach grundsätzlich nicht auf einen bestimmten Ableitungstyp beschränkt ; z. B.

(A) mit nominaler Nisbe : ntrr.j hr..w «göttlich an Erscheinungen»;

(B) mit präpositionaler Nisbe : hnt.j s.t «vorn in Bezug auf den Sitz » (dazu gleich Genaueres).

Die von Nomina abgeleiteten Nisben beinhalten im Gegensatz zu den in Abschnitt 1 behandelten, von Präpositionen abgeleiteten Relationsnisben keine Relation (allgemeine Lagebestimmung), sondern eine Qualität. Beispielsweise ist nicht alles, was in einer beliebigen Beziehung zu einem Gott steht, ntr.j, sondern nur das, was dem Wesen des Gottes entspricht, was göttliche Qualität hat. Die von Nomina abgeleiteten Nisben heben sich samt und sonders als solche Qualitätsnisben von den abstrakteren Relationsnisben ab.

Auch die von Präpositionen abgeleiteten Nisben, die in der präpositionalen Relation des Abschnitts 1 als Relationsnisben bezeichnet werden können, sind in der limitierenden Relation (Beispiel B) als Qualitätsnisben einzustufen und mit den von Nomina abgeleiteten auf

für Phonetik und allgemeine Sprachwissenschaft, 1 (1947), 85-108 ; ders., "'Konversion' und 'analytische' Tendenz in der ägyptischen Sprachentwicklung ", ebd. 4 (1950), 41-56) stark vermehrt und verschiedene "synthetische " Relationen zurückdrängt. 
eine Stufe zu stellen. Sie haben eine Qualität zum Inhalt, keine blosse Relation mehr. Z. B. ist ein hnt.j s.t " einer mit erst $\mathrm{r}$ a $\mathrm{ng}$ i g e m Sitz ", nicht etwa schlechthin "einer mit $\mathrm{x}$-beliebigem Sitz, der nur v o r n ist». Das "Vornsein» der Relationsnisbe reicht nicht zur Inhaltsbestimmung aus. Nicht jeder, der "seinen Sitz vorn hat " ist gleich ein hnt.j s.t, sondern nur der, bei dem das "Vornsein 》 einem Rang gleichkommt $\left.{ }^{(}\right)$. Ähnlich ist das zum Substantiv erstarrte hft.j "Feind 》, das in Abschnitt 3 noch einmal aufzuführen ist, nicht jedes "Gegenüber ", sondern der "Gegner ». Die räumliche Relation, die ursprünglich allein zugrunde gelegen haben mag, ist auf qualitativ bestimmte Umstände eingeschränkt.

Ich halte dafür, die Nisben nach ihrer Funktion einzuteilen. "Relationsnisben ", die immer von Präpositionen abgeleitet sind, sind solche, die in die präpositionale Relation eingehen ; "Qualitätsnisben 》, die sowohl von Nomina wie von Präpositionen abgeleitet sein können, sind solche, die in der limitierenden Relation (Limitation) stehen.

$\mathrm{Zu}$ der Regel, dass Nisben in der limitierenden Relation (Limitation) immer eine Qualität beinhalten, nicht aber eine blosse Relation (Lageangabe), gibt es relikthafte Ausnahmen, die zeigen, dass die Regel nicht schon immer galt. Die in den Grammatiken behandelten Verbindungen einer von einer Präposition abgeleiteten Nisbe mit einem Substantiv fallen inhaltlich in zwei Gruppen. So bedeutet die Verbindung der von der Präposition $(j) m$ abgeleiteten Relationsnisbe jm.j mit einem Substantiv $X, j m . j X$,

(C) meist soviel wie $n . t j m \mathrm{X}$ «der, welcher in $\mathrm{X}$ ist, der in $\mathrm{X} \mathrm{Be}-$ findliche »;

(D) seltener aber auch soviel wie n.tj X jm.f « der, in dem $X$ ist » $\left(^{2}\right)$.

Beide Verständnisweisen ergeben sich ungezwungen aus der Struktur der Relationsnisbe, daraus nämlich, dass die Wortwurzel eine Präposition ist, das ganze Wort aber durch die Ableitung in die Klasse der "Adjektive " überführt ist. So wie beispielsweise ein von einem Verb abgeleitetes und in die Klasse der "Adjektive " eingereihtes Partizip als verbaler Nukleus wie als nominaler Nukleus dient $\left({ }^{3}\right)$, so kann

(1) In das ägyptische hnt.j s.t eine reine Relationsnisbe hineinzulesen, hätte einen humoristischen Effekt, der eben darauf beruhte, dass man an der Sprachnorm vorbeiginge. Z. B. könnte man von einem armen Schlucker wohl einmal sagen, er sei ein hhnt.j s.t, weil er zufällig weit vorn sitzt. Das nur als Vermutung.

(2) Vgl. Edel, AäG § 370.

(3) Vgl. $s \underline{d} m s w$ " der ihn hört " neben $s d m n b$ " jeder, der hört 》. 
zunächst auch die von einer Präposition abgeleitete Relationsnisbe einerseits präpositionaler Nukleus (Konstruktion C) sein, andererseits " adjektivischer " Nukleus (Konstruktion D) ${ }^{1}$ ). Konstruktion C wurde oben in Abschnitt 1 als präpositionale Relation bestimmt. Konstruktion D lässt sich als "adjektivischer " Nukleus mit "bestimmendem Substantiv " erläutern. So wie ntr.j hr.w heisst "göttlich, vielmehr seine Erscheinungen sind göttlich », so heisst entsprechend jm.j $X$ «darinbefindlich, vielmehr $\mathrm{X}$ ist darinbefindlich ».

Nun gibt es allerdings für die gleichwertige Ausbildung der beiden Konstruktionsweisen $\mathrm{C}$ und D eine entscheidende Erschwernis dadurch, dass beide formal, auch im Satzakzent $\left({ }^{2}\right)$, zusammenfallen. Die unterschiedliche Art der syntaktischen Relation ist somit nicht explizit gegeben $\left(^{3}\right)$, sondern nur unterschoben. Die Schwierigkeit wurde in der Sprachgeschichte so gelöst, dass die Bedeutung D aufgegeben wurde. In der uns überlieferten Sprache kommen Belege noch in den bewahrenden religiösen Texten vor, sonst nur in erstarrten Resten $\left(^{4}\right)$.

Zwei Belege der Grammatiken scheinen der Deutung der Konstruktion D als limitierender Relation zu widersprechen, der erste, indem er ein Variantenverhältnis zu einem sog. indirekten Genitiv vortäuscht und deshalb die Deutung der Konstruktion D als sog. direkten Genitivs suggeriert; im zweiten hängt scheinbar vom Substantiv ein Suffixpronomen $a b$, das in der limitierenden Relation ausgeschlossen ist.

a) E. Edel liest ein Hntj-nj-jrtj $\left(^{(5)}\right.$ als Variante des Gottesnamens Hntj-jrtj « der vorn zwei Augen hat 》 $\left.{ }^{6}\right)$. Es gibt aber keine Möglichkeit, von einem sog. indirekten Genitiv aus im Altägyptischen zu der Bedeutung zu kommen, die man dem Namen geben müsste. Wenn die Verbindungen Nisbe + Substantiv in der Bedeutung C durch Nisbe + $n \cdot j+$ Substantiv ersetzt werden, so ist das etwas ganz anderes. Wie

(1) Es ist nicht richtig, beide Konstruktionen als "Verbindungen zwischen Nisbeformen von Präpositionen und dem von der Präposition regierten Substantiv " einzustufen (so Edel, AäG § 370). Die Bedeutung D ist aus "präpositionalen " Syntagmen nicht ableitbar.

(2) Nach den Fechtschen Regeln in jedem Fall 1 Kolon; s. G. Fecht, ZÄS 91 (1964), 34 (I) und 32 (C 4); MDIK 19 (1963), 68 (I) und 66 (C 4).

(3) Es sei denn in sehr speziellen Faktoren der Besetzung der beiden Planstellen oder des Kontexts, die das andere Verständnis ausschliessen.

(4) S. Erman, ZÄS 52 (1914), 107 f. ; ders., ÄG A $^{4} 231$ b; Edel, AäG § 370.

(5) Pyr. 601 e (T.).

(6) Edel, AäG § 370. 
man auch die Namen des «sehenden» und des «blinden Gottes» auflösen mag, in dem Pyramidenbeleg kann kein sog. indirekter Genitiv stecken.

b) Das merkwürdige Wort jm.j-rn.f "Liste », das aus einem Syntagma der Bedeutung $\mathrm{D}$ entstanden sein muss, enthält ein scheinbar regelwidriges Suffixpronomen, dann nämlich, wenn man als ursprünglichen Sinn versteht "Das, in dem sein Name ist » $\left(^{(1}\right)$. Dieses Verständnis scheitert daran, dass in der Liste ja weder der Name des vorher genannten Ganzen (s e i n Name) steht, noch der Name eines einzelnen Bestandstücks, sondern eben eine Liste von Namen. Setzt man dagegen als Ausgangsform das syntaktisch einwandfreie *jm.j-rn ohne Suffixpronomen an, so ergibt sich eine plausible Ableitung. *jm.j-rn "Das, in dem der Name (der Bestandstücke) steht » wäre bereits früh zu einem W o r t mit der Bedeutung "Liste » erstarrt. Man hängt nun erst ein Suffix an, das sich auf das vorhergehende Ganze bezieht: jmj-rn.f "seine Liste". Man mag unterstellen, dass das Suffixpronomen noch wechseln konnte und erst in einer erneuten Erstarrung zu einem W o r t jmj-rnf auf $f$ beschränkt wurde. Dass diese gesamte Verbindung als e i n Wort verstanden wurde, zeigt das ebenso wie jmj-rnf von der 12. Dynastie an belegte jmj-rnf $j r . j$ "List davon ". Die Ableitung ist hypothetisch, weil das Wort erst in der 12. Dynastie und dann bereits auf der letzten Entwicklungsstufe belegt ist. Da schon im Alten Reich die Syntagmen aus Nisbe + Substantiv in der Bedeutung D in der lebendigen Sprache keine Rolle spielen, hat man reichlich Platz, diese stufenweise Umdeutung unterzubringen. - Nur der Kuriosität halber sei erwähnt, dass die (äthiopischen) Sprachkenner der Spätzeit das Wort wieder in seine Bestandteile zerlegen und aus einem vermeintlichen jm.j-rn.f "Das, in dem sein Name steht " konsequenterweise ein sinnvolleres jm.j-rn.sn "Das, in dem ih re (d.h. der Einzelglieder des Ganzen) Namen stehen 》 machen $\left(^{2}\right)$.

\section{3. "Substantivierte» Qualitätsnisben in Genitivrelation.}

Nisben, die ihrer Fuṇktion nach "Adjektive" sind, können, wie die "Adjektive» weithin, "substantiviert» werden $\left({ }^{3}\right)$. Z. B. hft.j "Feind»;

(1) Vgl. Erman, $\mathrm{ÄG}^{4} \S 231$ b.

(2) Kawa IX, 61 f. ; 66 (Zeit: Aman-nēte-yerike).

(3) Kennzeichen der "Substantivierung " ist der Verlust der Genusvariabilität, Ergebnis, dass die Nisben zu einer Funktionsgemeinschaft "Substantiv " gehören, deren grösste Mitgliedermenge die Substantive der Formenlehre ausmachen. 
tp.j "Erstgeborener ". Von "substantivierten" Relationsnisben, d. h. solchen Nisben, die in präpositionaler Relation einen Satelliten nach sich haben (oben Abschnitt 1), soll nicht weiter die Rede sein; ihre Fähigkeiten als Nukleus bleiben unverändert auf die präpositionale Relation beschränkt. "Substantivierte» Qualitätsnisben dagegen verhalten sich nicht anders als andere Substantive und können in allen Relationen stehen, in denen diese vorkommen; z. B. als Nukleus im sog. indirekten Genitiv oder als Nukleus einer attributiven Relation (Nisbe + "Adjektiv »).

Interessant ist hier nur der sog. direkte Genitiv ${ }^{(1)}$. Einmal ist festzuhalten, dass Nisben durchaus als Nukleus der Genitivrelation stehen können. Zum andern zeigt sich, dass diese Konstruktion eine andere Bedeutung hat als die oben als präpositionale Relation erklärte, die bisweilen unzutreffend als Genitivrelation bezeichnet wurde. Beispiele : tp.j jtj.f "Erstgeborener seines Vaters»; tp.j rnp.t "Erster (Tag) des Jahres ». tp.j jtj.f, isoliert genommen, heisst neben "Erstgeborener seines Vaters 》 natürlich auch mit Relationsnisbe in präpositionaler Relation "Der auf seinem Vater (Befindliche)». Nur der Kontext entscheidet, welche Bedeutung tp.j zu unterlegen ist : die relationale oder die qualitative. Bei tp.j rnp.t dürfte durch den Sprachgebrauch die relationale Bedeutung von vornherein ausgeschieden oder doch sehr unwahrscheinlich sein; doch ist in entsprechend einengendem Kontext auch die andere denkbar. Bei der Ausscheidung der limitierenden Relation ("Adjektiv》 + bestimmendes Substantiv) hilft im Falle von $t p . j$ jtj.f bereits das Suffixpronomen, bei tp.j rnp.t wieder der Sprachgebrauch bzw. der Kontext.

Nur in solchen Fällen wie den hier behandelten ist es sinnvoll, die Verbindung einer Nisbe mit einem Substantiv als "Genitiv " zu bezeichnen.

\section{Nisben in mehr als e in er Relation.}

Die Einteilung der Nisben in Relationsnisben und Qualitätsnisben bezieht sich auf ihre Funktion, nicht auf den Wortkörper. Eine Relationsnisbe ist, solange sich die Bedeutungen des Wortes in den beiden Funktionen noch nicht $\mathrm{zu}$ sehr auseinanderentwickelt haben $\left(^{2}\right)$, das

(1) Belegt wohl nur noch in der ältesten Sprache. Später steht der sog. indirekte Genitiv, was am "substantivischen " Nukleus selbst nichts ändert.

(2) hft.j in h̆ft.j-hrr "gegenüber" (Wb. III, 276, 6) ist, auch nach Ausweis der 
gleiche Wort wie die gleichlautende Qualitätsnisbe. Beide Funktionen können miteinander verschränkt sein. So hängt $z$. B. von der «substantivierten " Nisbe $t p . j$ gleichzeitig ein Substantiv in präpositionaler Relation ab ( $t p . j=$ Relationsnisbe) und ein Suffixpronomen in Genitivrelation $(t p . j=$ Qualitätsnisbe $): t p . j w . s n$ t 3 «ihre auf Erden Befindlichen, ihre Hinterbliebenen 》 $\left.{ }^{1}\right)$. Der Unterschied zwischen Relationsnisbe und Qualitätsnisbe zeigt sich auch hier aufs schönste. Die Relation zwischen $t p . j w$ und $t_{3}$. gibt lediglich Auskunft über die abstrakte, räumliche Lage; die Relation zwischen $t p . j$ und.$s n$ dagegen bezeichnet mit tp.j nie und nimmer nur ein in bestimmtem räumlichem Verhältnis Vorfindliches, sondern die besonderen Qualitäten des "Besessenen », gleichgültig, ob man die Verbindung als tp.jw- $t$; "Nachkommen " + .sn « ihre 》 auflöst oder schon in tp.jw "Daraufbefindliche» + .sn "ihre " ein bedeutungsgesättigtes Syntagma sieht.

Wulfyarg SCHENKEL

Determinierung, für den Ägypter zweifellos nicht mehr dasselbe Wort wie $h f t . j$ "Feind", das zum Substantiv erstarrt ist und so bis ins Koptische fortlebt, dem die Nisbebildung verlorengegangen ist.

(1) S. Edel, AäG $\S 371$; weiter : Gardiner, $\mathrm{EG}^{3} \S 80$. 